\title{
A Highly Convenient Procedure for Oligodeoxynucleotide Purification
}

\author{
Durga Pokharel and Shiyue Fang*
}

Department of Chemistry, Michigan Technological University, 1400 Townsend Drive, Houghton, MI 49931, USA

\begin{abstract}
Purification of synthetic oligodeoxynucleotides (ODNs) is simply achieved by capping failure sequences with a polymerizable phosphoramidite followed by polymerization. In this article, the reduction of the amount of polymerization monomer to drastically increase ODN extraction efficiency, the use of a centrifugal filterunit to ease the extraction process and the notification of using fresh phosphoramidite solutions are described. In addition, further evidence to support the purity of ODN and discussions of ODN stability under radical polymerization conditions are provided.
\end{abstract}

Keywords: Catching by polymerization, DNA stability, high throughput, large scale, oligonucleotide, purification, radical.

\section{INTRODUCTION}

Synthetic oligodeoxynucleotides (ODNs) have found wide applications in areas such as molecular biology, synthetic biology and antisense drug development [1]. Their syntheses have been automated. Large scale and high throughput syntheses are both possible [2-6]. During synthesis, the first nucleoside is attached to a solid support. Subsequent nucleosides are added sequentially to give the full-length ODN. The coupling reactions are highly efficient, but cannot be $100 \%$, which inevitably generates failure sequences. These failure sequences are usually capped with acetic anhydride to ease purification. At the end of synthesis, the ODN is cleaved from support and fully deprotected. The product contains failure sequences and small molecules besides full-length sequences, and has to be purified for most applications and ideally for all applications. The small molecules are easy to remove because they are neutral and ODNs are anionic. Removal of failure sequences is, however, more difficult. Currently, the most widely used method is HPLC. Drawbacks include high cost of instrument and column, labor intensiveness, and high waste to product ratio. The method is difficult to automate, expensive to scale up, and unsuitable for high throughput purification. Other methods have been developed to solve the problems [7-12], but still have various shortcomings [13].

We recently reported a catching failure sequences by polymerization method for ODN purification [13, 14]. In this method, the failure sequences are capped with a poly merizable phosphoramidite such as $\mathbf{1}$ (Fig. 1). Purification is achievedby copolymerization with $N, N$-dimethylacrylamide. The failure sequences are incorporated into polymer. The full-length sequences and small molecules remainin solution and polymer matrix. Extraction with water and $n \mathrm{BuOH}$ precipitation gives pure ODN. In this letter, we report several highly important refinements of the technology.

*Address correspondence to this author at the Department of Chemistry, Michigan Technological University, 1400 Townsend Drive, Houghton, MI 49931, USA; Tel: +1 906487 2023; Fax: +1 906487 2061;

E-mail: shifang@mtu.edu
In our initial procedure, large excess of polymerization monomer was used. One refinement is to reduce this amount. This refinement drastically increased the extraction efficiency of ODN from polymer. Previously, extraction of ODN was accomplished by pipetting supernatant, which was inconvenient. The second refinement is to carry out polymerization in a centrifugal filter unit and to perform extraction by simple spins. Besides these refinements, further evidences to prove the purity of ODN, and the explanation of the stability of ODN under radical polymerization conditions are provided.

The greatly simplified procedure is demonstrated by the purification of the 20-mer ODN 5'-TCA TTG CTG CTT AGA CCG CT-3' (2). The ODN synthesis procedure, which includes capping failure sequences by $\mathbf{1}$, was similar as previously described [14]. Briefly, the synthesizer manufacturer (ABI 394) suggested $1 \mu$ mol DNA synthesis cycle was copied to create a new cycle. The steps for capping with acetic anhydride were replaced with those for capping with $\mathbf{1}$, which were achieved by delivering 1 and activator to column. The delivery method was the same as the coupling steps except that an additional delivery was added $(2.5$ seconds $\times 3)$ and after each delivery a wait of 30 seconds was added. Following capping, three washes (acetonitrile to column, 10 seconds; reverse flush, 6 seconds) were added. The synthesis was set up as usual with $1(0.1 \mathrm{M}$ in acetonitrile) [14] being placed in bottle 5. 4,5Dicyanoimidazole $(0.25 \mathrm{M}$ in acetonitrile) was used as activator. Because the catching by polymerization method can isolate full-length ODN from very complex mixture, we lowered the concentration of phosphoramidite monomer (Pac-dA, acetyl-dC, 4-isopropyl-Pac-dG, dT) solutions from the usually used $0.1 \mathrm{M}$ to $0.05 \mathrm{M}$ (in acetonitrile). At the end of synthesis, detritylation was performed. Cleavage and deprotection were carried out with concentrated $\mathrm{NH}_{4} \mathrm{OH}$ at room temperature on synthesizer $(15 \mathrm{~min} \times 4$, then $2 \mathrm{~h}$ ). The solution was divided into four portions and dried in 1.5 centrifugal tubes. One portion was dissolved in $600 \mu 1$ water, $20 \mu \mathrm{l}$ was injected into HPLC to give the crude profile (trace A, Fig. 2). 


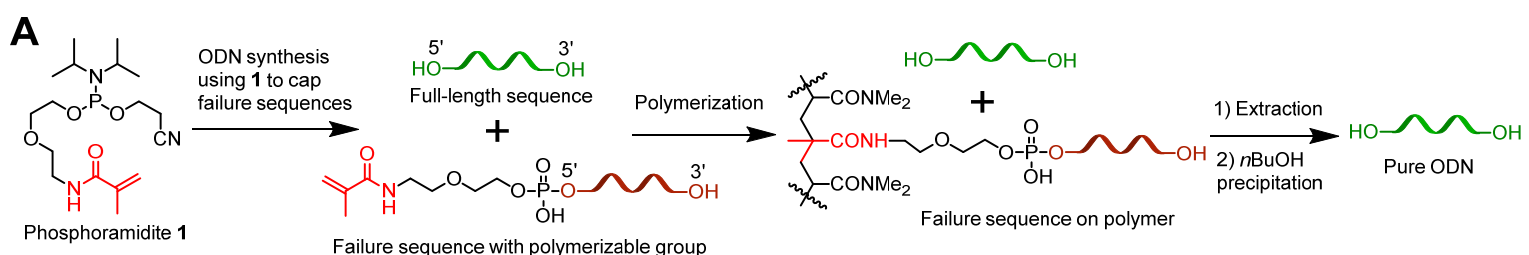

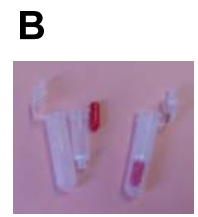

Centrifugal filter units

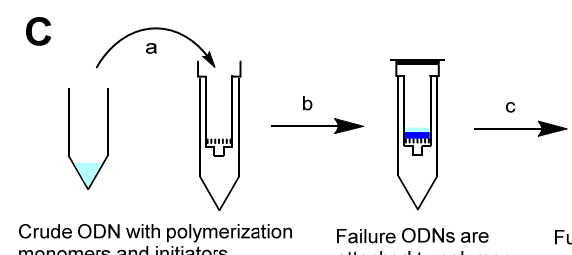
monomers and initiators attached to polyme

Full-length ODN and small molecules in filtrate

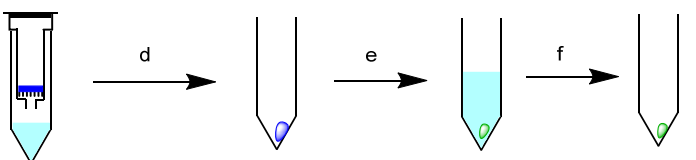

Full-length ODN with small molecules
ODN precipitated Pure ODN by $n \mathrm{BuOH}$

Fig. (1). The catching failure sequences by polymerization ODN purification technology. (A) Working principle. (B) Picture of centrifugal filter units. (C) Workflow: (a) To crude ODN in a centrifugal tube are added polymerization monomer, cross-linker and initiators. After mixing, the contents are transferred to the upper tube of a centrifugal filter unit. (b) The cap is closed and the mixture is allowed to polymerize for $1 \mathrm{~h}$. (c) The gel is cut into several pieces and the supernatant is collected in the lower tube by spin. To the gel was added water. After standing at room temperature for $10 \mathrm{~min}$, spin again. The extraction is repeated two more times. (d) The upper tube is removed, and the filtrate is evaporated to dryness. (e) To the residue is added concentrated $\mathrm{NH}_{4} \mathrm{OH}$. The cap is closed, and the mixture is warmed to 80 ${ }^{\circ} \mathrm{C}$ for $15 \mathrm{~min}$ in a heating block. After cooling to room temperature, $n \mathrm{BuOH}$ is added. The mixture is vortexed and then spun for 2 min. (f) The supernatant is removed by a pipette.
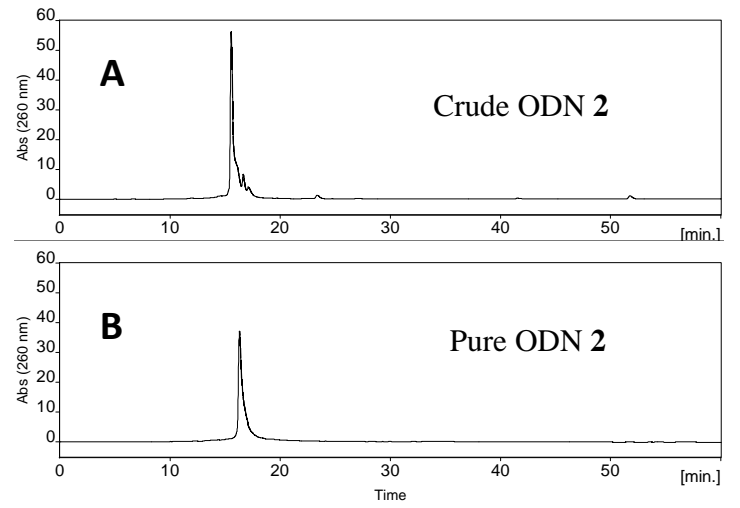

Fig. (2). HPLC profiles of ODN 2. Conditions: column, C-18, $5 \mu \mathrm{m}, 100 \AA$, $250 \times 3.20 \mathrm{~mm}$; solvent A, $0.1 \mathrm{M}$ triethylammonium acetate, $5 \%$ acetonitrile; solvent B, $90 \%$ acetonitrile; gradient, solvent B (0\%-45\%) in solvent A over 60 min; flow rate, $0.5 \mathrm{~mL} / \mathrm{min}$; detection, $260 \mathrm{~nm}$.

Another portion of the crude $2(\sim 0.25 \mu \mathrm{mol})$ was dissolved in $50 \mu \mathrm{l}$ water. Short vortex and spin were performed to ensure complete dissolution and bring down solution to bottom. To the solution was added $6 \mu l$ polymerization solution $\left(0.37 \mathrm{M} \quad N, N^{\prime}\right.$-methylenebisacrylamide and $3.7 \mathrm{M} \mathrm{N}, N$-dimethylacrylamide) followed by a short vortex and spin. The molar ratio of the polymerization monomer over ODN is $\sim 100 / 1$. The initiators $\left(\mathrm{NH}_{4}\right)_{2} \mathrm{~S}_{2} \mathrm{O}_{8}(5 \%, 5 \mu \mathrm{l})$ and TMEDA $(0.66 \mathrm{M}, 5 \mu \mathrm{l})$ were then added. After a short vortex and spin, the mixture was transferred to the center of the filter in a centrifugal filter unit (2 $\mathrm{ml}$, pore size 7-20 $\mu \mathrm{m}$, Aldrich) immediately in one portion (Fig. 1) [15]. It is suggested to close the bottom of the tube, but we found that the solution would not leak even without closing. Care needs to be taken to transfer all contents and to avoid splashing, which is easily achievable by manipulating the pipette with a steady force as opposed to abrupt sucking and pushing. The unit was closed and the mixture was allowed to polymerize for 1 hour. The gel was cut into about four pieces, and the bottom of the upper tube was opened. The unit was spun for about 30 seconds, which separated the supernatant from gel. To the upper tube was added $50 \mu \mathrm{l}$ water, which should cover all the gel. After standing for 10 minutes, the unit was spun. The extraction was repeated two more times. The supernatants were evaporated to dryness. To the residue, $100 \mu \mathrm{l}$ concentrated $\mathrm{NH}_{4} \mathrm{OH}$ was added, and the mixture was heated to $80{ }^{\circ} \mathrm{C}$ for 15 minutes on a heating block (CAUTION: safety goggles and face shield are needed for potential explosion). After cooling to room temperature, $900 \mu \mathrm{l} n \mathrm{BuOH}$ was added. The mixture was vortexed shortly and spun for 2 minutes. The supernatant was removed with a pipette. The white flake was pure ODN. To determine purity and recovery yield, it was dissolved in $600 \mu \mathrm{l}$ water, and $20 \mu \mathrm{l}$ was injected into HPLC to give trace B (Fig. 2). The ODN was $100 \%$ pure. The recovery yield was estimated to be $97 \%$ by dividing the area of the peak at 15.5 minutes in trace B by that in trace A.To see if more ODN could be obtained from the gel, the extraction and precipitation process was repeated. HPLC analysis did not show any ODN (supplementary data), which 
indicated that three extractions in a time as short as 30 minutes were sufficient. We also performed a blank control experiment in which no ODN was used. We did not see any residue. HPLC analysis did not found any UV active material.

To confirm that the ODN peak in trace B does not contain any failure sequences, we synthesized the 19-mer ODN5'-CATTGCTGCTTAGACCGCT-3' (3), which was identical to 2 except that the $20^{\text {th }}$ base was not added. The ODN was purified by catching by polymerization. Coinjection of $\mathbf{2}$ and $\mathbf{3}$ gave a broadened peak. When a slower gradient was used, the two were well-resolved. With the same slower gradient, 2 did not show any 19-mer failure sequence (supplementary data). Because 19-mer failure sequence is most difficult to resolve from the full-length 20mer sequence, it is conclusive that all failure sequences can be removed by the catching by polymerization purification technique. Another potential impurity in $\mathbf{2}$ may be the one from oxidation of $\mathrm{dG}$ to 8 -oxo-dG, and this could not be detected by HPLC. We had described the synthesis of the same 20-mer sequence of 2 with one dG being replaced with 8-oxo-dG 5'-TCA TTG CT(8-oxo-dG) CTT AGA CCG CT$3^{\prime}(4)$, and its digestion to nucleosides. We found that 8-oxo$\mathrm{dG}$ could be detected by HPLC. In contrast, 8 -oxo-dG could not be detected from nucleosides from digesting 2 [14]. In addition, we had subjected the $G$ nucleoside into the radical polymerization conditions and found that it was stable [16]. Here, we provide further evidence that $\mathrm{dG}$ is stable under radical acrylamide polymerzation conditions. When ODN 4, which contains 8-oxo-dG, was exposed to concentrated $\mathrm{NH}_{4} \mathrm{OH}$ at elevated temperature, multiple peaks were observed in HPLC profile (supplementary data). This observation indicated that ODNs containing 8-oxo-dG is unstable under such conditions. Because our purification procedure involves in heating ODN in concentrated $\mathrm{NH}_{4} \mathrm{OH}$, and HPLC analysisshowed single peak, we can conlude that ODNs purified with the catching by polymerization technology do not contain 8-oxo-dG.

It is interesting to note that radicals can damage DNA [17]. Then, why is ODN stable under the radical acrylamide polymerization conditions? To anwser this question, we need to consider the fact that DNA damage is mostly caused by the highly reactive hydroxyl radical. This radical is so reactive that it breaks carbon-hydrogen bonds with little selectivity. The radical used to initiate the acrylamide polymerization reaction is the sulfate radical, which was reported to be 3,000 times less reactive than the hydroxyl radical [18]. In addition, under the polymerization conditions used for purification, large excess of acrylamide is used. This acrylamide could serve as a radical scavenger like vitamin $\mathrm{E}$ in the biological system. Once the sulfate radical is formed in the initiation stage of polymerization, it is quickly converted to the carbon radical of acrylamide. This radical is stabilized by the amide group through resonance, and may not be reactive enough to damage ODN, which requires abstraction of hydrogen atom from a carbonhydrogen bond or breaking aromaticity of nucleoside bases by adding a radical to an aromatic ring.

For using the catching by polymerization method for ODN purification, one very important lesson we learned in the past few years is that fresh nucleoside phosphoramidite solutions need to be used for ODN synthesis. Otherwise, the purity of product may be lower. For example, to reduce costs, in some of our previous experiments, phosphoramidite solutions that were stored in a freezer under nitrogen in jars containing Drierite over half a year were used. The ODNs purified by catching by ploymerization were found contaminated with small amount of unidentifiable impurities [14]. After realizing this, we always used phosphoramidite solutions prepared within one week and found that ODN purity was consistently $100 \%$ after purification by polymerization. Leaving the solutions on the synthesizer for one week was found having no adverse effects on the purification results. It should be noted that the phosphoramidites do not need to be freshly purchased. In fact, the ones we used were usually stored in a freezer for more than six months.

The above refinements including using less acrylamide polymerization solution, using a centrifugal filter unit, and using fresher phosphoramidite solutions are critical for the catching by polymerization purification technology to be practically useful. The resulted improvements are summarized in Table 1. For example, in previous procedure, we used large excess of polymerization monomer. Therefore, we had to extract $0.25 \mu \mathrm{mol}$ ODN from more than $500 \mu \mathrm{l}$ gel $[13,14]$. In the improved procedure, we only used $6 \mu 1$ monomer solution and the gel volume is less than $20 \mu \mathrm{l}$. Importantly, we observed supernatant over the gel, which may contain a large portion of ODN. In contrast, using previous procedure, there was only one phase. Due to the reduced gel volume and the existence of supernatant, the efficiency of ODN extraction is drastically improved. Previously, we had to be very careful to suck up the supernatant by pipette during extraction [13, 14]. It was inconvenient because gel fragments occasionally block pipette tip, and fine fragments can be sucked up, which contaminate ODN. With a centrifugal filter unit, extraction can be carried out by short spins.

In conclusion, several very important refinements of the catching failure sequences by polymerization ODN purification technology are presented. These refinements are critical for the technology to be practically useful. In addition, to demonstrate the purity of ODN, we synthesized a 19-mer failure sequence and proved that HPLC can resolve it from the 20-mer full-length sequence. The observation of ODN that contains 8 -oxo-dG is unstable under the purification conditions further confirmed that ODN is stable under the acrylamide radical polymerization conditions. The reasons for the stability are discussed. With these new results and findings, we are now very confident that the catching by polymerization purification method is highly convenient and highly reliable. We expect that it be widely used for small scale, large scale, and high throughput purification.

\section{CONFLICT OF INTEREST}

S.F. is the inventor of two US patents (one issued, one pending) on the technology filed by Michigan Technological University. 
Table 1. Summary of advantages of improved procedure over previous procedure.

\begin{tabular}{|c|l|l|l|}
\hline Entry & \multicolumn{1}{|c|}{ Items } & \multicolumn{1}{|c|}{ Previous Procedure } & \multicolumn{1}{|c|}{ Improved Procedure } \\
\hline \hline 1 & Polymerization solution & $250 \mu 1$ & $6 \mu 1$. Save monomers and reduce gel volume \\
\hline 2 & ODN solution & $250 \mu 1$ & $50 \mu 1$ \\
\hline 3 & Gel volume & $>500 \mu l$. Larger volume makes extraction less efficient & $<20 \mu 1$. Smaller volume makes extraction more efficient \\
\hline 4 & Supernatant & No. All ODN in gel, difficult to extract & Yes. Most ODN in supernatant, easier to extract \\
\hline 5 & Water for extraction & $12 \mathrm{ml}(3 \mathrm{ml} \times 4)$. Needs more time to evaporate & $0.15 \mathrm{ml}(50 \mu 1 \times 3)$. Needs less time to evaporate \\
\hline 6 & Extraction time & $12 \mathrm{~h}(3 \mathrm{~h} \times 4)$. Cannot finish in one day & 30 min $(10$ min $\times 3)$ \\
\hline 7 & & $\begin{array}{l}\text { Pipetting supernatant. Difficult due to tip blockage by } \\
\text { gel. Each extraction takes } \sim 20 \text { min. Not suitable for } \\
\text { high throughput purification }\end{array}$ & $\begin{array}{l}\text { Spin. Each extraction takes } 10 \text { sec. Suitable for high } \\
\text { throughput purification }\end{array}$ \\
\hline 8 & Extract & $\begin{array}{l}\text { May contain gel, which needs additional removal and } \\
\text { reduces recovery yield }\end{array}$ & No gel fragments, and very clean \\
\hline 9 & Recovery yield & $70-95 \%$ & $>95 \%$ \\
\hline 10 & ODN Purity & $92-100 \%$ & $100 \%$. Due to fresher phosphoramidite solution \\
\hline
\end{tabular}

\section{ACKNOWLEDGEMENTS}

Financial support from US NSF (CHE-0647129 and CHE-1111192), MTU Biotech Research Center, MUCI, and MTU REF-TC; the assistance from Mr. Dean W. Seppala (electronics) and Mr. Jerry L. Lutz (NMR); and NSF equipment grants (CH-1048655 and $\mathrm{CHE}-9512445)$ are gratefully acknowledged.

\section{SUPPLEMENTARY MATERIAL}

Supplementary material is available on the publisher's web site along with the published article.

\section{REFERENCES}

[1] Singh, Y.; Murat, P.; Defrancq, E. Recent developments in oligonucleotide conjugation. Chem. Soc. Rev., 2010, 39(6), 20542070.

[2] Tian, J.D.; Ma, K.S.; Saaem, I. Advancing high-throughput gene synthesis technology. Mol. Biosyst., 2009, 5(7), 714-722.

[3] Hughes, R.A.; Miklos, A.E.; Ellington, A.D. Gene synthesis: Methods and applications. Synth Biol., Part B, 2011, 498, 277-309.

[4] Ma, S.Y.; Tang, N.; Tian, J.D. DNA synthesis, assembly and applications in synthetic biology. Curr. Opin. Chem. Biol., 2012, $16(3-4), 260-267$.

[5] Abramova, T. Frontiers and approaches to chemical synthesis of oligodeoxyribonucleotides. Molecules, 2013, 18(1), 1063-1075.

[6] Kosuri, S.; Church, G.M. Large-scale de novo DNA synthesis: Technologies and applications. Nat. Methods, 2014, 11(5), 499507.

[7] Fang, S.; Bergstrom, D.E. Reversible biotinylation phosphoramidite for 5'-end-labeling, phosphorylation, and affinity purification of synthetic oligonucleotides. Bioconjug. Chem., 2003, 14(1), 80-85.
[8] Beller, C.; Bannwarth, W. Noncovalent attachment of nucleotides by fluorous fluorous interactions: Application to a simple purification principle for synthetic DNA fragments. Helv. Chim. Acta., 2005, 88(1), 171-179.

[9] Fang, S.; Bergstrom, D.E. Fluoride-cleavable biotinylation phosphoramidite for 5 '-end-labeling and affinity purification of synthetic oligonucleotides. Nucleic Acids Res., 2003, 31(2), 708715 .

[10] Sproat, B.S.; Rupp, T.; Menhardt, N.; Keane, D.; Beijer, B. Fast and simple purification of chemically modified hammerhead ribozymes using a lipophilic capture tag. Nucleic Acids Res., 1999, 27(8), 1950-1955.

[11] Pearson, W.H.; Berry, D.A.; Stoy, P.; Jung, K.Y.; Sercel, A.D. Fluorous affinity purification of oligonucleotides. J. Org. Chem., 2005, 70(18), 7114-7122.

[12] Fang, S.; Bergstrom, D.E. Reversible 5'-end biotinylation and affinity purification of synthetic rna. Tetrahedron Lett., 2004 45(43), 7987-7990.

[13] Fang, S.; Fueangfung, S.; Lin, X.; Zhang, X.; Mai, W.; Bi, L.; Green, S.A. Synthetic oligodeoxynucleotide purification by polymerization of failure sequences. Chem. Commun., 2011, 47(4), 1345-1347.

[14] Pokharel, D.; Yuan, Y.; Fueangfung, S.; Fang, S. Synthetic oligodeoxynucleotide purification by capping failure sequences with a methacrylamide phosphoramidite followed by polymerization. $R S C A d v$., 2014, 4, 8746-8757.

[15] Polymerization in the tube over the filter was found less efficient. This problem can be solved by rinsing the upper tube in a solvent such as ethanol overnight or washing three times.

[16] Fang, S.; Fueangfung, S. Scalable synthetic oligodeoxynucleotide purification with use of a catching by polymerization, washing, and releasing approach. Org. Lett., 2010, 12(16), 3720-3723.

[17] Breen, A.P.; Murphy, J.A. Reactions of oxyl radicals with DNA. Free Radic Biol. Med., 1995, 18(6), 1033-1077.

[18] Liang, C.J.; Su, H.W. Identification of sulfate and hydroxyl radicals in thermally activated persulfate. Ind. Eng. Chem. Res., 2009, 48(11), 5558-5562.

(C) Pokharel and Fang; Licensee Bentham Open.

This is an open access article licensed under the terms of the Creative Commons Attribution Non-Commercial License (http://creativecommons.org/licenses/ by-nc/3.0/) which permits unrestricted, non-commercial use, distribution and reproduction in any medium, provided the work is properly cited. 